\title{
The SHiP experiment at CERN
}

\author{
Walter M. Bonivento, on behalf of the SHiP Collaboration ${ }^{1, a}$ \\ ${ }^{1}$ Istituto Nazionale di Fisica Nucleare, Sezione di Cagliari, Cittadella Universitaria di Monserrato, Monserrato \\ (CA), Italy
}

\begin{abstract}
The discovery of the Higgs boson has fully confirmed the Standard Model of particles and fields. Nevertheless, there are still fundamental phenomena, like the existence of dark matter and the baryon asymmetry of the Universe, deserving an explanation that could come from the discovery of new particles. Searches for new physics with accelerators are performed at the LHC, looking for high massive particles coupled to matter with ordinary strength. A new experiment at CERN meant to search for very weakly coupled particles in the few $\mathrm{GeV}$ mass domain has been recently proposed. The existence of such particles, foreseen in different theoretical models beyond the Standard Model, is largely unexplored. A beam dump facility using high intensity $400 \mathrm{GeV}$ protons is a copious source of such unknown particles in the GeV mass range. The beam dump is also a copious source of neutrinos and in particular it is an ideal source of tau neutrinos, the less known particle in the Standard Model. The neutrino detector can also search for dark matter through its scattering off the electrons. We report the physics potential of the SHiP experiment.
\end{abstract}

\section{Introduction}

The discovery of the Higgs boson is certainly a big triumph of the Standard Model. Nevertheless, there are several phenomena deserving an explanation that the Standard Model is unable to provide: the existence of dark matter and its nature, the baryonic asymmetry of the Universe and neutrino masses. It is therefore clear that new physics is there and presumably several new particles have still to be discovered. Searches for new physics with accelerators are being carried out at the LHC, especially suited to look for high mass particles with ordinary couplings to matter. Complementary searches for very weakly coupled and therefore long lived particles require a beam dump facility. Such a facility is made of a high density proton target, followed by a hadron stopper and a muon shield. Apart from residual muons, the only remaining particles are electron, muon and tau neutrinos on top of hidden, long lived particles produced either in proton interactions or in secondary particle decays.

A new experiment, Search for Hidden Particles (SHiP), has been proposed [1], designed to operate at a beam dump facility to be built at CERN and to search for weakly coupled particles in the few GeV mass range. Since a high intensity tau neutrino flux is produced by such a facility from $D_{s}$ decays, the experimental apparatus foresees a neutrino detector to study the tau neutrino cross-section and discover the tau anti-neutrino. This detector would also be suited to detect dark matter through the

\footnotetext{
a e-mail: walter.bonivento@ca.infn.it
} 


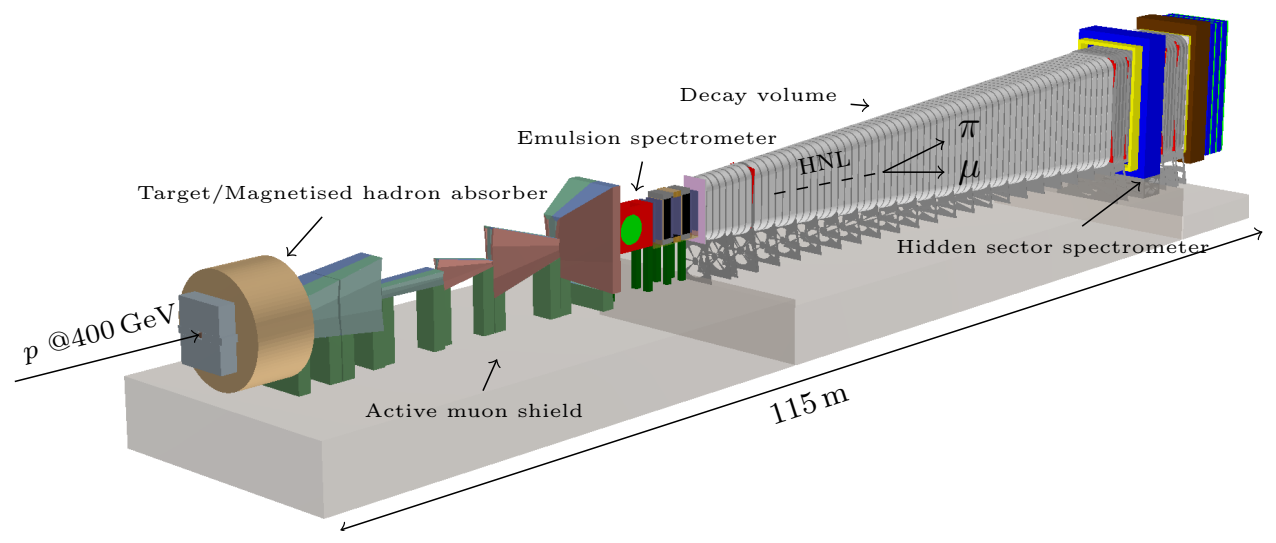

Figure 1. The beam dump facility and the SHiP detector.

scattering off the atomic electrons of its target. The physics case for such an experiment is widely discussed in Ref. [2]. We will review here the physics potential of this experiment for a selection of physics channels.

\section{The SHiP facility and apparatus}

In five years, the facility will integrate $2 \times 10^{20} 400 \mathrm{GeV}$ protons, produced by the SPS accelerator complex, impinging on a 12 interaction length $\left(\lambda_{\text {int }}\right)$ target made of Molybdenum and Tungsten, followed by a $30 \lambda_{\text {int }}$ iron hadron absorber. Hidden particles in the $\mathrm{GeV}$ mass range would be produced mostly by the decay of charmed hadrons produced in proton interactions. $D_{s}$ mesons, copiously produced among charmed hadrons, are a source of tau neutrinos through their fully leptonic decay. Therefore, the SHiP facility is ideal also to study the physics of tau neutrinos, the less known particle in the Standard Model.

Figure 1 shows the SHiP facility to be placed in the North Area: downstream of the target, the hadron absorber filters out all hadrons, therefore only muons and neutrinos are left. An active muon shield is designed with two sections with opposite polarity to maximize the muon flux reduction: it reduces the muon flux from $\sim 10^{10}$ down to $\sim 10^{5}$ muons per spill. $4 \times 10^{13}$ protons are extracted in each spill, designed to be $1 \mathrm{~s}$ long to reduce the detector occupancy. A first successful test of the SPS cycle with a 1s long spill was performed in April 2015.

An emulsion spectrometer to detect tau neutrinos and search for dark matter is located downstream of the muon shield, followed by the decay vessel and the detector for hidden particles. The Collaboration has been requested by the SPSC Committee to prepare a Comprehensive Design Report by 2018, in the framework of the Physics Beyond Colliders working group, launched in March 2016 at CERN. This working group is meant to prepare proposals as input for the High Energy panel of the European Strategy to be held in 2019-2020; approval of the experiment will follow the recommendation of this panel. The construction and installation will last until the third long shutdown of the LHC and the data taking is assumed to start in 2026.

The emulsion spectrometer detector is made of a magnetised target region, followed by a muon spectrometer. The neutrino target is based on the emulsion cloud chamber technology employed by the OPERA experiment [3], with a compact emulsion spectrometer, made of a sequence of very low density material and emulsion films to measure the charge and momentum of hadrons in magnetic 
field. Indeed, this feature would allow to discriminate between tau neutrinos and anti-neutrinos also in the hadronic decay channels of the tau lepton. The emulsion target is complemented by high resolution tracking chambers to provide the time stamp to the event and connect muon tracks from the target to the muon spectrometer. The muon spectrometer is based on the concept developed for the OPERA apparatus: a dipolar iron magnet where drift tubes provide the momentum and resistive plate chambers provide the tracking within the iron slabs. The atoms of the emulsion target would also act as the target of dark matter coming from the decays of the dark photons produced from the proton interaction in the target.

The detector for hidden particles is located in the downstream part of a $60 \mathrm{~m}$ long evacuated decay vessel, with a rectangular transverse section of $5 \times 10 \mathrm{~m}^{2}$, with the longer axis vertically. The hidden particles are supposed to decay within the vessel. The requirement to have less than 1 neutrino interaction in the vessel over five years sets the pressure to about 1 mbar. A magnetic spectrometer is located downstream of the vessel: it is made of straw tubes with a material budget of $0.5 \% X_{0}$ per station, achieving a position resolution of $120 \mu \mathrm{m}$ per straw, with 8 hits per station on average. This gives a momentum resolution of about $1 \%$. The vessel would be surrounded by a liquid scintillator layer to tag particles coming from outside. Downstream of the spectrometer, an hadronic and electromagnetic calorimeter and a muon filter are meant for particle identification. A timing detector with a resolution better than 100 ps makes the combinatorial background negligible.

\section{Search for hidden particles}

Extensions of the Standard Model in the low mass region foresee the existence of particles as singlets with respect to the Standard Model gauge group. These particles couple to different singlet composite operators (so-called Portals) of the Standard Model. The neutrino can be considered as the first example of a hidden particle: it was postulated in 1930 to explain the puzzle of the beta decay, it is very light and extremely weakly interacting. The SHiP detector located immediately downstream of a beam dump has the potentiality to discover all of them, since it is sensitive to very weakly interacting and long lived particles in a wide unexplored range of their masses and couplings. We describe in the following its sensitivity to dark photons, heavy neutral leptons and dark matter. For the sensitivity to the other portals, we refer to Ref. [1].

\subsection{Search for hidden photons}

Dark photons are vectorial particles coupled to the photon with a lagrangian term $L=-k B_{\mu \nu} V^{\mu \nu} / 2$. In a beam dump facility, they can be produced directly from the proton interaction with the target and in the decay of particles like $\pi^{0}, \eta, \omega$ and $\eta^{\prime}$. The dark photon may decay into dark matter particles or, if no lighter hidden particles exist, into final states like $e^{+} e^{-}, \mu^{+} \mu^{-}$or $q \bar{q}$. Recently, data from old experiments have been reanalysed and exclusion plots have been derived together with cosmological limits $[4,5]$. The left plot of figure 2 shows the exclusion limits at $90 \%$ C.L. set by SHiP in case no signal is found. The parameters displayed are the mass and $\varepsilon^{2}$ squared coupling of the dark photon. It is worth noting that the region investigated by SHiP covers a new region, unexplored by previous searches.

\subsection{Search for heavy neutral leptons}

Neutrino masses are explained by the see-saw mechanism with a lagrangian term given by

$$
L={ }_{\imath} \bar{N}_{I} \partial_{\mu} \gamma^{\mu} N_{I}-Y_{I \alpha} \bar{N}_{I}^{C} \tilde{H} L_{\alpha}-M_{I} \bar{N}_{I}^{C} N_{I}+\text { h.c. }
$$



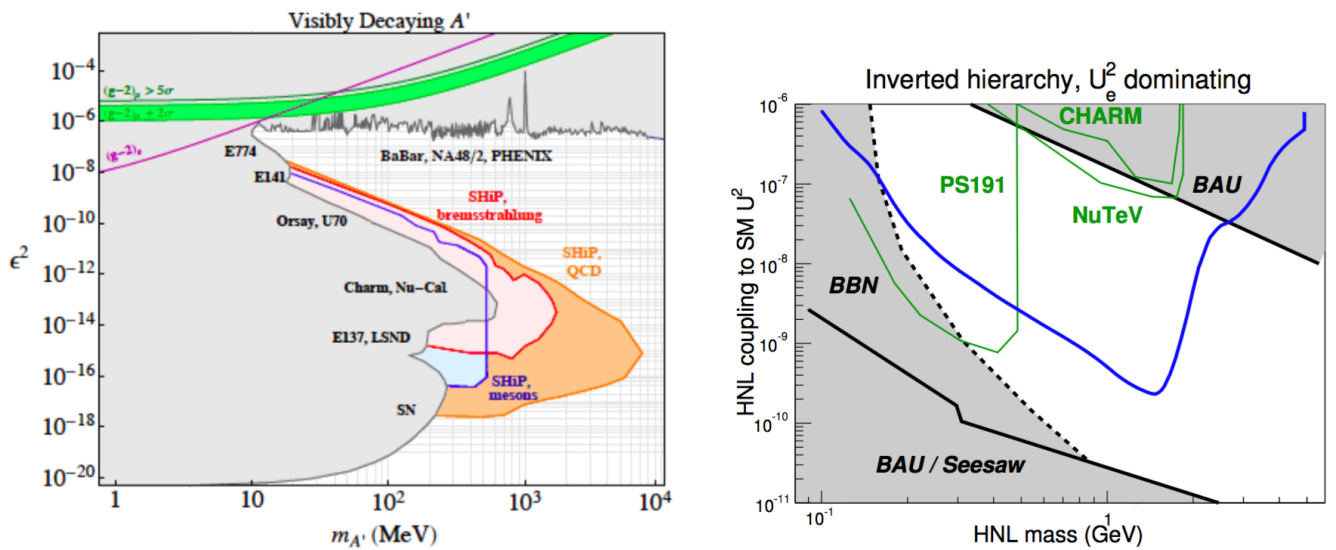

Figure 2. Left: exclusion limit at $90 \%$ C.L. in the dark photon search, in the plane defined by its mass and $\epsilon^{2}$ squared coupling. Right: exclusion limits at $90 \%$ C.L. in the heavy neutral lepton search, for inverted hierarchy with $U_{e}^{2}$ dominating, according to Ref. [11].

where 3 right-handed heavy neutrinos $N_{I}$ are introduced. The mass of active neutrinos is given by $m_{v} \simeq m_{D}^{2} / M_{I}$ where $m_{D} \simeq Y_{I \alpha} v$ and $v \simeq 246 \mathrm{GeV}$ is the Higgs vacuum expectation value.

If several conditions are satisfied, among which that $\mathrm{CP}$ is not conserved in the leptonic sector, these right-handed neutrinos could also explain the leptogenesis and threfore the baryogenesis $[6,7]$. Such models do not claim to explain also dark matter: a dark matter candidate could be provided by a fourth right-handed neutrino or an axion. Moreover they do not require two of the three heavy neutrinos to be quasi-degenerate because $\mathrm{CP}$ violation does not need to be enhanced by mass degeneracy.

In the attempt of explaining also dark matter with three heavy neutrinos, the so-called $v \mathrm{MSM}[8,9]$ was first introduced in 2005, followed by more refined versions [10] later on. The role of the three right-handed neutrinos is different as well as their mass: $M_{1}$ could be a dark matter candidate with the mass of several $\mathrm{keV}$ while $M_{2}$ and $M_{3}$ should be states with a mass of about $1 \mathrm{GeV}$ and quasidegenerate to provide the baryon asymmetry of the Universe. The states $N_{2}$ and $N_{3}$ could be produced in the decay of sufficiently massive particles like charmed hadrons. These states would be long lived particles that in turn could decay, for example, into a pion-muon pair.

The right plot of Figure 2 shows the experimental and cosmological bounds on the search for heavy neutral leptons. It also reports superimposed the exclusion limits at $90 \%$ C.L. SHiP could set in case no signal is found, assuming the model reported in Ref. [11] with inverted hierarchy and a dominant electron coupling, $U_{e}^{2}: U_{\mu}^{2}: U_{\tau}^{2}=48: 1: 1$. The parameter space explored by SHiP extends in the cosmologically relevant region that is experimentally unexplored.

\section{Physics with the neutrino detector}

The tau neutrino is the less known particle in the Standard Model. The observation of this particle was finally confirmed in 2008 when 9 candidates events were reported with an estimated background of 1.5 [12]. In the same paper, the tau neutrino cross-section, with a $50 \%$ uncertainty due to the small statistical sample and to the rather poor knowledge of the incoming flux, was also reported. The DONUT experiment was unable to separate neutrinos from anti-neutrinos. The OPERA experiment has detected five tau neutrinos [13-17], discovering the tau neutrino appearance from muon neutrino 
oscillations. The only leptonic decay observed by OPERA [15] shows negative charge as expected from a $v_{\tau}$ interaction. Therefore, so far there is no direct evidence for tau anti-neutrinos.

The number of $v_{\tau}$ and $\bar{v}_{\tau}$ emerging from the molybdenum target can be estimated as:

$$
N_{v_{\tau}+\bar{v}_{\tau}}=4 N_{p} \frac{\sigma_{c \bar{c}}}{\sigma_{p N}} f_{D_{s}} \operatorname{Br}\left(D_{s} \rightarrow \tau\right)=6.6 \times 10^{15}
$$

where $N_{p}$ is the number of interacting protons, $\sigma_{c \bar{c}}=18.1 \pm 1.7 \mu$ barn [18] is the associated charm production per nucleon, $\sigma_{p N}=10.7$ mbarn is the hadronic cross-section per nucleon in a Mo target, $f_{D_{s}}=\left(8.8 \pm 0.6_{-0.9}^{+0.5}\right) \%$ [19] is the fraction of $D_{s}$ mesons produced, $\operatorname{Br}\left(D_{s} \rightarrow \tau\right)=(5.54 \pm 0.24) \%$ [20] is the $D_{s}$ branching ratio into $\tau$ and the factor 4 accounts for the charm pair production and for the two $v_{\tau}$ produced per $D_{s}$ decay. The SHiP facility is therefore a $v_{\tau}$ factory.

Given the neutrino target mass of 9.6 tons, the neutrino flux, the geometrical acceptance of the detector and the standard model cross-section [21], one expects about 7000 interactions of tau neutrinos and 3500 of tau anti-neutrinos. The charged-current $v_{\tau}\left(\bar{v}_{\tau}\right)$ differential cross-section is given by five structure functions. The contribution to the cross-section of $F_{4}$ and $F_{5}$ structure functions [22] is negligible in muon and electron neutrino interactions due to the light charged lepton mass. On the contrary, given the non-negligible mass of the $\tau$ lepton, tau neutrino scattering is sensitive to their contribution. Indeed, the hypothesis of $F_{4}=F_{5}=0$ would significantly increase the $v_{\tau}$ and $\bar{v}_{\tau}$ charged-current deep-inelastic cross sections and the corresponding number of expected $v_{\tau}$ and $\bar{v}_{\tau}$ interactions. SHiP is sensitive to a non-null value of $F_{4}$ and $F_{5}$ [1].

\subsection{Strange parton distribution}

Charmed hadrons are produced in neutrino and anti-neutrino charged-current interactions at the level of about 5\%. Advantages of the nuclear emulsion technology in the detection of neutrino-induced charm production are discussed in Ref. [23]. Despite the fact that $1.280 .000 v_{\mu}$ and $270.000 \bar{v}_{\mu}$ charged-current interactions were collected by the NuTeV/CCFR Collaboration, only $5102 v_{\mu}$ and $1458 \bar{v}_{\mu}$ events were identified as charm production induced by neutrino charged-current interactions. The largest number of events in an emulsion experiment was collected by CHORUS with 2013 charm candidates from $v_{\mu}$ and only 32 from $\bar{v}_{\mu}$ [24]. SHiP will integrate about $10^{5}$ charm candidates, more than one order of magnitude larger than the present statistics, with a large $(\sim 30 \%)$ contribution from anti-neutrinos. Charm production in neutrino scattering is extremely sensitive to the strange quark content of the nucleon, especially with anti-neutrinos where the $s$-quark is dominant. SHiP will improve significantly the uncertainty on the strange quark distribution in the nucleon as shown in the left plot of Figure 3 in terms of $s^{+}=s(x)+\bar{s}(x)$ in the $0.02<x<0.35$ range.

\subsection{Dark Matter search}

The dark matter at an accelerator can be produced through the decay of a dark photon and it can thus be copiously produced at a beam dump facility. Given the proton energy, a dark matter in the GeV and sub-GeV mass range can be produced at SHiP. Direct searches performed in the underground laboratories rely on the observation of nuclear recoils induced by the scattering of dark matter. Given the non-relativistic nature of dark matter, the recoils induced show a kinetic energy of the keV order if the dark matter mass is around $1 \mathrm{GeV}$. This makes the search for dark matter at around $1 \mathrm{GeV}$ or below extremely difficult. The sensitivity of current experiments in that range is therefore limited by the capability of observing such low energy recoils. At the accelerator, on the contrary, the dark matter would be ultra-relativistic and it could be observed through its scattering off the electrons of 

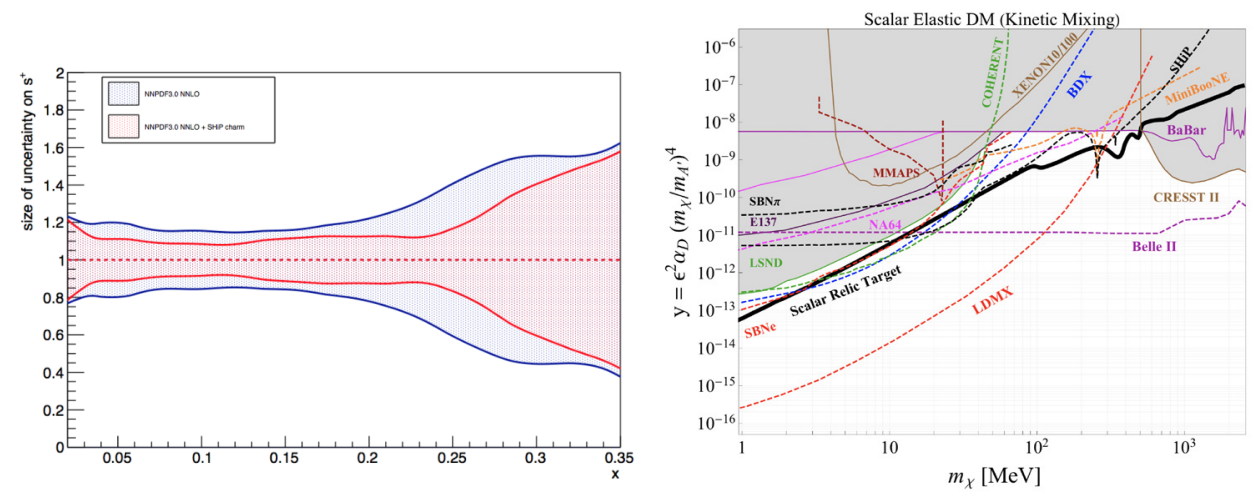

Figure 3. Left: improvement of the accuracy on $s^{+}$with SHiP (red) compared to the present status (blue) in the $0.02<x<0.35$ range. Right: sensitivity to the light dark matter (figure from [25])

the lead-emulsion target of the neutrino detector. The elastic interaction of dark matter particles with electrons produces one electron in the final state, thus mimicking elastic interaction of neutrinos that constitute the main background for this search. The total background to this search is estimated in Ref. [1] and the right plot of Figure 3 shows the sensitivity in the plane of the coupling $y$ and the dark matter mass $m_{\chi}$. In the same plot, the sensitivity of other experimental programs is shown together with the relic dark matter density. SHiP sensitivity is such to test in some mass regions the predictions made from the relic dark matter density.

\section{References}

[1] M. Anelli et al., arXiv:1504.04956.

[2] S. Alekhin et al., Rept. Prog. Phys. 79 (2016) no.12, 124201.

[3] N. Agafonova et al., OPERA Coll., JINST 4 (2009) P04018.

[4] J. Brunner, Phys. Lett. B731 (2014) 320.

[5] A. Fradette et al., Phys. Rev. D90 (2014) 035022.

[6] M. Drewes, et al., JHEP 1303 (2013) 096.

[7] M. Drewes, Int. J. Mod. Phys. E22 (2013) 1330019.

[8] T. Asaka and M. Shaposhnikov, Phys. Lett. B620 (2005) 17.

[9] M. Shaposhnikov, Nucl. Phys. B763 (2007) 49.

[10] L. Canetti et al., Phys. Rev. Lett. 110 (2013) 061801.

[11] L. Canetti and M. Shaposhnikov, JCAP 1009 (2010) 001.

[12] K. Kodama et al., Phys. Rev. D78 (2008) 052002.

[13] N. Agafonova et al., Phys. Lett. B691 (2010) 138.

[14] N. Agafonova et al., JHEP 1311 (2013) 036.

[15] N. Agafonova et al., Phys. Rev. D89 (2014) 051102.

[16] N. Agafonova et al., PTEP 2014 (2014) 101C01.

[17] N. Agafonova et al., Phys. Rev. Lett. 115 (2015) 121802.

[18] C. Lourenco, H.K. Wohri, Phys. Rept. 433 (2006) 127.

[19] H. Abramowicz et al., JHEP 1309 (2013) 058. 
[20] K.A. Olive et al., Chin. Phys. C38 (2014) 090001.

[21] M. H. Reno, Phys. Rev. D 74 (2006) 033001.

[22] C. H. Albright and C. Jarlskog, Nucl. Phys. B 84 (1975) 467.

[23] G. De Lellis et al., Physics Reports 399 (2004) 227.

[24] A. Kayis-Topaksu et al., New J. Phys. 13 (2011) 093002.

[25] M Battaglieri et al., arXiv:1707.04951 